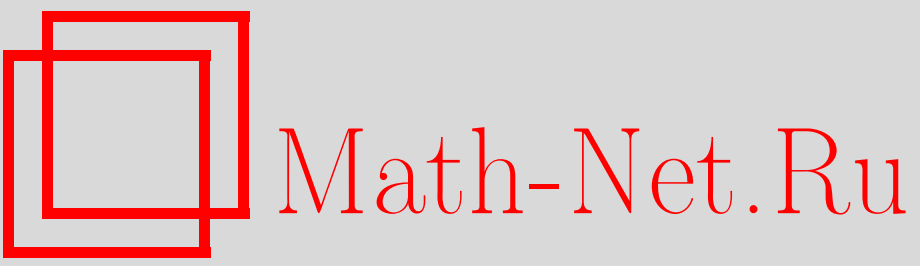

В. Р. Коган, Дисперсия лагранжевых траекторий в случайном крупномасштабном поле скорости, ТМФ, 2000, том 122, номер 3, 456-467

DOI: https://doi.org/10.4213/tmf580

Использование Общероссийского математического портала Math-Net.Ru подразумевает, что вы прочитали и согласны с пользовательским соглашением

http://www.mathnet.ru/rus/agreement

Параметры загрузки:

IP: 54.81 .137 .203

26 апреля 2023 г., 15:39:52 
ТЕОРЕТИЧЕСКАЯ

И МАТЕМАТИЧЕСКАЯ

ФИЗИКА

Том 122, № 3

март, 2000

(C) 2000 r.

В.Р. Коган*

\section{ДИСПЕРСИЯ ЛАГРАНЖЕВЫХ ТРАЕКТОРИЙ В СЛУЧАЙНОМ КРУПНОМАСШТАБНОМ ПОЛЕ СКОРОСТИ}

Изучается статистика расстояния $R(t)$ между двумя лагранжевыми траекториями в пространственно-гладком турбулентном поле скорости с произвольным временем корреляции и с негауссовым распределением. В этой ситуации имеются два безразмерных параметра: $\alpha$ - степень отличия от гауссовой статистики, и $\beta=\tau D$, где $\tau-$ время корреляции скорости, а $D$ - характерный градиент скорости. Асимптотически $R(t)$ имеет логнормальную статистику, которую можно характеризовать средней скоростью разбегания $\bar{\lambda}$ и дисперсией $\Delta$. Использована техника большой размерности пространства $d$ для оценки величин $\bar{\lambda}$ и $\Delta$ при различных значениях параметров $\alpha$ и $\beta$. В работе [1] было продемонстрировано, что $\bar{\lambda} \sim D$ при $\beta \ll 1$ и $\bar{\lambda} \sim \sqrt{D / \tau}$ при $\beta \gg 1$. При этом оценка $\Delta$ не универсальна и зависит от деталей парного коррелятора скорости. С корреляторами скорости высшего порядка связан дополнительный вклад в дисперсию $\Delta$, который может быть оценен как $\alpha D^{2} \tau$ при $\beta \ll 1$ и $\alpha \beta / \tau$ при $\beta \gg 1$. Если $\alpha$ превышает некоторое критическое значение $\alpha_{\mathrm{cr}}$, значения $\bar{\lambda}$ и $\Delta$ определяются высшими неприводимыми корреляторами градиента скорости и наша схема перестает работать. Это критическое значение может быть оценено как $\alpha_{\text {cr }} \sim \beta^{-1}$ при $\beta \ll 1$ и $\alpha_{\text {cr }} \sim \beta^{-1 / 2}$ при $\beta \gg 1$.

\section{1. ВВЕДЕНИЕ}

Дисперсия (закон разбегания) лагранжевых траекторий, т.е. траекторий частиц, движущихся со скоростью жидкости, является классическим объектом изучения в физике турбулентности. Эта фундаментальная проблема, восходяшая к Ричардсону [2], имеет также большое практическое значение (например, для описания структуры облаков). Именно на языке лагранжева формализма следует выражать скейлинговые соотношения для статистики турбулентного потока, замаскированные эффектом сильного сноса $[3,4]$. Заметим также, что дисперсия лагранжевых траекторий связана со статистикой пассивного скаляра, переносимого турбулентным полем скорости $[5,6]$. Поэтому весьма важно установить связь между статистикой турбулентной скорости и законом разбегания лагранжевых траекторий. Строгий вывод закона разбегания был сделан только в предположении короткого времени корреляции скорости и гауссового распределения $[7,8]$.

\footnotetext{
${ }^{*}$ Институт теоретической физики им. Л. Д. Ландау РАН, Москва, Россия
} 
Представляет интерес выйти за рамки этих предположений. Это оказывается возможным при исследовании поведения лагранжевых траекторий на расстояниях, меньших, чем вязкая длина, где поле скорости незначительно изменяется от точки к точке. При этом дисперсия лагранжевых траекторий определяется распределением градиентов скорости (так как однородный снос вообще не меняет расстояния между лагранжевыми частицами), и можно говорить о движении лагранжевых частиц в крупномасштабном поле скорости. Но даже в этом случае сложно исследовать произвольную статистику градиентов скорости. Однако нас больше интересуют качественные заключения, касающиеся влияния различных факторов, характеризуюших статистику скорости, на дисперсию лагранжевых траекторий. Как было показано в работе [1], для этой цели весьма полезным оказывается использование техники большой размерности пространства $d$, которая позволяет получать заключения, справедливые и при $d \sim 1$. Мы будем изучать статистику расстояний между двумя лагранжевыми частицами $R(t)$, считая, что это расстояние фиксировано в начальньй момент времени: $R(t=0)=R_{0}$. Траектория лагранжевой частицы $\varrho(t)$ определяется уравнением

$$
\partial_{t} \varrho=\mathbf{v}(t, \varrho)
$$

где $\partial_{t} \equiv \partial / \partial t$ и $\mathbf{v}-$ скорость жидкости. Скорость будет считаться случайной функцией времени и координат. Следуя Крайчнану [5], можно считать ее статистику гауссовой с нулевым средним и парным коррелятором

$$
\left\langle v_{\alpha}\left(t_{1}, \mathbf{r}_{1}\right) v_{\beta}\left(t_{2}, \mathbf{r}_{2}\right)\right\rangle=\delta\left(t_{1}-t_{2}\right) V_{\alpha \beta}\left(\left|\mathbf{r}_{1}-\mathbf{r}_{2}\right|\right) \text {. }
$$

На расстояниях, малых по сравнению с крупномасштабной накачкой, для тензора $V_{\alpha \beta}$ предполагается следующее скейлинговое поведение:

$$
\begin{aligned}
V_{\alpha \beta}(\mathbf{r}) & =V_{0} \delta_{\alpha \beta}-K_{\alpha \beta}(r), \\
K_{\alpha \beta}(r) & \propto r^{2-\gamma} .
\end{aligned}
$$

Напомним, что в теории турбулентности, помимо масштаба упомянутой накачки, имеется еще малый масштаб, так называемая вязкая длина, на которой начинает эффективно действовать диссипация. На масштабах, меньших вязкой длины, это приводит к сглаживанию турбулентного поля скорости. Значение скейлингового показателя при этом $\gamma=0$. Эта ситуация замечательна тем, что при выполнении условий, приводящих к ней, скорость, как случайный процесс, является функцией только времени, а именно, вводя тензор градиентов скорости

$$
\sigma_{\alpha \beta}=\nabla_{\beta} v_{\alpha}
$$

можно показать, что

$$
v_{\alpha}(t, \mathbf{r})=r_{\beta} \sigma_{\alpha \beta}(t),
$$

причем $\sigma_{\alpha \beta}(t)$ - случайная функция времени [9]. В формуле (6) и ниже по повторяющимся индексам предполагается суммирование. Хотя в этом рассуждении считалось, что 
статистика скорости гауссова и декоррелирована во времени, все заключения являются справедливыми и в случаях, когда статистика негауссова и произвольно коррелирована, поскольку в рассуждении используется лишь тот факт, что коррелятор скорости $n$-го порядка содержит слагаемые, пропорциональные $r^{n}$. С физической точки зрения представление (6) оказалось возможным лишь постольку, поскольку поле скорости на субвязких масштабах оказывается гладким, с малыми градиентами.

Возврашаясь к динамике лагранжевых траекторий, можно показать, что вектор $\mathbf{R}=$ $\varrho_{1}-\varrho_{2}$, определяюший расстояние между двумя лагранжевыми частицами, в крупномасштабном поле скорости подчиняется уравнению

$$
\partial_{t} R_{\alpha}=\sigma_{\alpha \beta} R_{\beta}
$$

Заметим, что условие несжимаемости $\operatorname{div} \mathbf{v}=0$ записывается, как равенство нулю следа матрицы $\sigma_{\alpha \beta}$ :

$$
\sigma_{\alpha \alpha}=0 .
$$

Уравнение (7) можно переписать в виде двух уравнений

$$
\begin{gathered}
\partial_{t} n_{\alpha}=\sigma_{\alpha \beta} n_{\beta}-\sigma_{\gamma \beta} n_{\beta} n_{\gamma} n_{\alpha}, \\
\partial_{t} \ln R=\sigma_{\alpha \beta} n_{\beta} n_{\gamma},
\end{gathered}
$$

где $\mathbf{n}$ - единичный вектор в направлении $\mathbf{R}$. Мы видим, что статистика $\mathbf{n}$ не зависит от $R$. Тогда в силу центральной предельной теоремы из (9) следует, что на временах, бо́льших времени корреляции скорости $\tau$, величина $R$ имеет логнормальную статистику, т.е. функцию распределения вероятности для $R(t)$ можно записать в следуюшем виде:

$$
\mathcal{P}(t, R) \propto \exp \left\{-\frac{\left[\ln \left(R / R_{0}\right)-\bar{\lambda} t\right]^{2}}{2 \Delta t}\right\}
$$

где $\bar{\lambda}$ - средний темп разбегания траекторий, $\Delta$ - дисперсия этого темпа. Пользуясь формулой (10), можно найти асимптотические значения для корреляторов вида

$$
\left\langle R^{n}(t)\right\rangle=R_{0}^{n} \exp \left[n\left(\frac{n \Delta}{2}+\bar{\lambda}\right) t\right] .
$$

Это вычисление становится чрезвычайно полезным для корреляторов с $n=2$ и $n=4$, выражения для которых имеют вид

$$
\begin{aligned}
& \left\langle R^{2}(t)\right\rangle=R_{0}^{2} \exp [2(\Delta+\bar{\lambda}) t], \\
& \left\langle R^{4}(t)\right\rangle=R_{0}^{4} \exp [4(2 \Delta+\bar{\lambda}) t] .
\end{aligned}
$$

Полезность этих выражений становится очевидной, если найти следующий неприводимый коррелятор (что и является главным вычислительным результатом этой работы):

$$
\frac{\left\langle\left\langle R^{4}(t)\right\rangle\right\rangle}{\left\langle R^{2}(t)\right\rangle^{2}}=\frac{\left\langle R^{4}(t)\right\rangle-\left\langle R^{2}(t)\right\rangle^{2}}{\left\langle R^{2}(t)\right\rangle^{2}}=\exp (4 \Delta t)-1 .
$$


Итак, нашей основной задачей является вычисление величин $\bar{\lambda}$ и $\Delta$, входяших в (10). Для случая предельно долгокоррелированной скорости величина $\bar{\lambda}$ была найдена Бэтчелором [10], а для случая $\delta$-коррелированной скорости - Крайчнаном [8]. Ряд заключений о зависимости величины $\bar{\lambda}$ от времени корреляции скорости $\tau$ для простейшего вида гауссовой статистики скорости был сделан в работах [11, 12]. В работе [1], где использовался метод больших размерностей $d$ и статистика скорости предполагалась гауссовой, была исследована дисперсия $\Delta$ для различных значений $\tau$ и для разных скейлинговых законов зависимости корреляшионных функций скорости от времени. В настояшей работе, используя формализм работы [1], мы исследуем влияние отклонения статистики скорости от гауссовой, т.е. роль высших неприводимых корреляторов $\sigma_{\alpha \beta}$. Пока степень отличия от гауссовой статистики невелика, ее роль сводится к появлению дополнительного вклада в $\Delta$ и незначительной поправки к $\lambda$. При увеличении степени отклонения от гауссовой статистики дисперсия $\Delta$ по величине достигает $\bar{\lambda}$, после чего уже именно высшие корреляторы $\sigma_{\alpha \beta}$ будут определять как $\Delta$, так и $\bar{\lambda}$. K сожалению, наша техника не позволяет исследовать этот значительно отличающийся от гауссова режим, однако она позволяет установить, при каких значениях высших корреляторов он начинается при различных значениях $\tau$.

\section{2. ТЕХНИКА БОЛЬШИХ РАЗМЕРНОСТЕЙ}

В настоящем разделе кратко изложены процедура, использованная в [1], и основные результаты настояшей работы. При больших размерностях пространства $d$ вектор $\mathbf{R}$ имеет большое число компонент, поэтому имеет место эффект самоусреднения, позволяюший свести высшие корреляторы к парному $[13,14]$, например

$$
\left\langle R^{4}\right\rangle \approx\left\langle R^{2}\right\rangle^{2}
$$

Сравнение (15) с (12), (13) показывает, что это соотношение заведомо нарушается на больших временах или, другими словами, применимость техники больших размерностей $d$ ограничена временами $\Delta t \ll 1$. Необходимо также вспомнить, что равенства $(12),(13)$ являются асимптотическими при $t \gg \tau$. Таким образом, на время наблюдения $t$ техникой накладываются ограничения как сверху, так и снизу. Тем не менее при больших значениях $d$ имеется область перекрытия применимости (12), (13) и техники больших $d: \tau \ll t \ll \Delta^{-1}$, это условие предполагает неравенство $\tau \Delta \ll 1$, которое и реализуется при больших $d$. При таких временах из выражения (14) получаем

$$
\frac{\left\langle R^{4}\right\rangle-\left\langle R^{2}\right\rangle^{2}}{\left\langle R^{2}\right\rangle^{2}}=4 \Delta t
$$

Именно это выражение будет использоваться при вычислении дисперсии $\Delta$.

Ниже будет использоваться диаграммная техника Уайльда [15], обобшенная в работе [16] на широкий класс динамических систем (изложение этой техники можно найти в [17]). Точнее, будет использован порождающий эту диаграммную технику функциональный интеграл $[18,19]$. Правила обрашения с функциональным интегралом можно 
найти в [20]. В соответствии с результатами $[16,18,19]$ корреляционные функции векторного поля $\mathbf{R}$ могут быть записаны как функциональные интегралы по $\mathbf{R}(t)$ и вспомогательному полю $\overline{\mathbf{R}}(t)$ с весом, который определяется уравнением (7). Например, парньй коррелятор имеет вид

$$
\begin{gathered}
\left\langle\mathbf{R}\left(t_{1}\right) \mathbf{R}\left(t_{2}\right)\right\rangle=\int \mathcal{D} R \mathcal{D} \bar{R} \exp \left(i \mathcal{I}_{\text {eff }}\right) \mathbf{R}\left(t_{1}\right) \mathbf{R}\left(t_{2}\right), \\
\exp \left(i \mathcal{I}_{\text {eff }}\right)=\left\langle\exp \left\{i \int d t\left(i \bar{R}_{\alpha} \partial_{t} R_{\alpha}-i \bar{R}_{\alpha} \sigma_{\alpha \beta} R_{\beta}\right)\right\}\right\rangle_{\sigma}
\end{gathered}
$$

где мы ввели эффективное действие $\mathcal{I}_{\text {eff }}$ в соответствии с определением $(18)$, в котором $\langle\ldots\rangle_{\sigma}$ означает усреднение по статистике градиентов скорости $\sigma_{\alpha \beta}$. Коррелятор $\left\langle\mathbf{R}\left(t_{1}\right) \overline{\mathbf{R}}\left(t_{2}\right)\right\rangle$ имеет смысл восприимчивости системы, а потому обрашается в нуль при $t_{1}<t_{2}[1]$. Коррелятор же $\left\langle\overline{\mathbf{R}}\left(t_{1}\right) \overline{\mathbf{R}}\left(t_{2}\right)\right\rangle$ тождественно равен нулю.

В случае гауссовой статистики $\sigma_{\alpha \beta}$ усреднение в $(18)$ тривиально и приводит к следуюшему выражению для эффективного действия:

$$
i \mathcal{I}_{\text {eff }}=i \int d t \overline{\mathbf{R}} \partial_{t} \mathbf{R}-\frac{1}{2} \int d t_{1} d t_{2} \bar{R}_{\alpha}\left(t_{1}\right) R_{\beta}\left(t_{1}\right) \bar{R}_{\gamma}\left(t_{2}\right) R_{\mu}\left(t_{2}\right)\left\langle\sigma_{\alpha \beta}\left(t_{1}\right) \sigma_{\gamma \mu}\left(t_{2}\right)\right\rangle .
$$

В негауссовом случае в эффективное действие войдут также и высшие неприводимые корреляторы тензора $\sigma_{\alpha \beta}$, совокупность которых мы обозначим в виде выражения $i \mathcal{I}_{\text {high }}$. Парный коррелятор градиентов скорости в изотропном случае можно записать в следующем виде:

$$
\left\langle\sigma_{\alpha \mu}(t) \sigma_{\beta \nu}(0)\right\rangle=\frac{D}{d \tau}\left\{g\left(\frac{t}{\tau}\right)\left[\delta_{\alpha \beta} \delta_{\mu \nu}-\frac{1}{d} \delta_{\alpha \mu} \delta_{\beta \nu}\right]+\frac{1}{d} g_{1}\left(\frac{t}{\tau}\right)\left[\delta_{\alpha \beta} \delta_{\mu \nu}-\delta_{\alpha \nu} \delta_{\beta \mu}\right]\right\}
$$

где $g, g_{1}$ - произвольные функции времени, которые предполагаются нормированными условием

$$
\int_{-\infty}^{+\infty} d \xi g(\xi)=2
$$

Здесь мы перешли к безразмерному времени $\xi=t / \tau, \tau-$ время корреляции. Введем еше одну безразмерную постоянную

$$
\beta=D \tau
$$

Интересующие нас корреляционные функции получаются разложением по "внешним полям" $X, Y, Z$ производящего функционала:

$$
i \mathcal{I}_{x y z}=i \mathcal{I}_{\mathrm{eff}}+\int d \xi_{1} d \xi_{2}\left[X\left(\xi_{1}, \xi_{2}\right)\left(\overline{\mathbf{R}}_{1} \mathbf{R}_{2}\right)+Y\left(\xi_{1}, \xi_{2}\right)\left(\mathbf{R}_{1} \mathbf{R}_{2}\right)+Z\left(\xi_{1}, \xi_{2}\right)\left(\overline{\mathbf{R}}_{1} \overline{\mathbf{R}}_{2}\right)\right]
$$

Взяв логарифмические производные от функционала

$$
Z_{x y z}=\int \mathcal{D} \mathbf{R} \mathcal{D} \overline{\mathbf{R}} \exp \left(i \mathcal{I}_{x y z}\right)
$$


по полям $X, Y, Z$, получим необходимые корреляторы:

$$
\begin{aligned}
& F\left(\xi_{1}, \xi_{2}\right)=\frac{1}{d}\left\langle R_{\alpha}\left(\xi_{1}\right) R_{\alpha}\left(\xi_{2}\right)\right\rangle=\left.\frac{1}{d} \frac{\delta \ln Z_{x y z}}{\delta Y\left(\xi_{1}, \xi_{2}\right)}\right|_{0}, \\
& G\left(\xi_{1}, \xi_{2}\right)=\frac{1}{d}\left\langle\bar{R}_{\alpha}\left(\xi_{1}\right) R_{\alpha}\left(\xi_{2}\right)\right\rangle=\left.\frac{1}{d} \frac{\delta \ln Z_{x y z}}{\delta X\left(\xi_{1}, \xi_{2}\right)}\right|_{0} .
\end{aligned}
$$

Знак $\left.\right|_{0}$ означает, что производные вычисляются при $X=Y=Z=0$. Заметим, что в силу условия причинности функция $G\left(\xi, \xi^{\prime}\right)$ обрашается в нуль при $\xi^{\prime}<\xi$. Для задачи сушественны также и более обшие величины

$$
\begin{aligned}
\mathcal{F}\left(\xi_{1}, \xi_{2}\right) & =\frac{1}{d} \frac{\delta \ln Z_{x y z}}{\delta Y\left(\xi_{1}, \xi_{2}\right)} \\
\mathcal{G}\left(\xi_{1}, \xi_{2}\right) & =\frac{1}{d} \frac{\delta \ln Z_{x y z}}{\delta X\left(\xi_{1}, \xi_{2}\right)} \\
\overline{\mathcal{F}}\left(\xi_{1}, \xi_{2}\right) & =\frac{1}{d} \frac{\delta \ln Z_{x y z}}{\delta Z\left(\xi_{1}, \xi_{2}\right)}
\end{aligned}
$$

Последняя величина вследствие условия причинности равна нулю, если $X=Y=$ $Z=0[1]$. Уравнения на $\mathcal{F}, \mathcal{G}, \overline{\mathcal{F}}$ можно получить, основываясь на функциональных соотношениях вида

$$
\int \mathcal{D} \mathbf{R} \mathcal{D} \overline{\mathbf{R}} \frac{\delta}{\delta \bar{R}_{\alpha}}\left[R_{\alpha}\left(\xi^{\prime}\right) \exp \left(i \mathcal{I}_{x y z}\right)\right]=0 .
$$

Если аккуратно вычислить функциональные производные в соотношениях вида (28), то для всех написанных выше корреляторов можно получить следующие уравнения:

$$
\begin{aligned}
& i \partial_{\xi} \mathcal{F}\left(\xi, \xi^{\prime}\right)-\beta \int d \xi_{1} g\left(\xi-\xi_{1}\right) \mathcal{G}\left(\xi_{1}, \xi^{\prime}\right) \mathcal{F}\left(\xi_{1}, \xi\right)+ \\
& +\int d \xi_{1}\left[X\left(\xi, \xi_{1}\right) \mathcal{F}\left(\xi^{\prime}, \xi_{1}\right)+2 Z\left(\xi, \xi_{1}\right) \mathcal{G}\left(\xi_{1}, \xi^{\prime}\right)\right]=0, \\
& \delta\left(\xi-\xi^{\prime}\right)+i \partial_{\xi} \mathcal{G}\left(\xi^{\prime}, \xi\right)-\beta \int d \xi_{1} g\left(\xi-\xi_{1}\right) \overline{\mathcal{F}}\left(\xi_{1}, \xi^{\prime}\right) \mathcal{F}\left(\xi_{1}, \xi\right)+ \\
& \quad+\int d \xi_{1}\left[X\left(\xi, \xi_{1}\right) \mathcal{G}\left(\xi^{\prime}, \xi_{1}\right)+2 Z\left(\xi, \xi_{1}\right) \overline{\mathcal{F}}\left(\xi^{\prime}, \xi_{1}\right)\right]=0, \\
& \delta\left(\xi-\xi^{\prime}\right)-i \partial_{\xi} \mathcal{G}\left(\xi, \xi^{\prime}\right)-\beta \int d \xi_{1} g\left(\xi-\xi_{1}\right) \overline{\mathcal{F}}\left(\xi_{1}, \xi\right) \mathcal{F}\left(\xi_{1}, \xi^{\prime}\right)+ \\
& \quad+\int d \xi_{1}\left[X\left(\xi_{1}, \xi\right) \mathcal{G}\left(\xi_{1}, \xi^{\prime}\right)+2 Y\left(\xi_{1}, \xi\right) \mathcal{F}\left(\xi_{1}, \xi^{\prime}\right)\right]=0, \\
& -i \partial_{\xi} \overline{\mathcal{F}}\left(\xi, \xi^{\prime}\right)-\beta \int d \xi_{1} g\left(\xi-\xi_{1}\right) \overline{\mathcal{F}}\left(\xi_{1}, \xi\right) \mathcal{G}\left(\xi^{\prime}, \xi_{1}\right)+ \\
& \quad+\int d \xi_{1}\left[X\left(\xi_{1}, \xi\right) \overline{\mathcal{F}}\left(\xi_{1}, \xi^{\prime}\right)+2 Y\left(\xi_{1}, \xi\right) \mathcal{G}\left(\xi^{\prime}, \xi_{1}\right)\right]=0 .
\end{aligned}
$$

Эти уравнения написаны в главном порядке по $d$. Учет всех членов сильно загромождает эти уравнения, делая невозможным их исследование. Более того, в таком точном виде, как нетрудно видеть, они перестают быть замкнутыми, преврашаясь в бесконечную 
цепочку взаимосвязанных уравнений, подобную цепочке Боголюбова в теории кинетических уравнений.

Для получения коррелятора $F\left(\xi_{1}, \xi_{2}\right)$ необходимо просто положить $X=Y=Z=0$. Для получения дисперсии необходимо рассмотреть корреляторы

$$
\begin{aligned}
F\left(\xi, \xi^{\prime}, \xi^{\prime \prime}\right)= & \frac{1}{d}\left\langle R_{\alpha}(\xi) R_{\alpha}\left(\xi^{\prime}\right) R^{2}\left(\xi^{\prime \prime}\right)\right\rangle- \\
& -\frac{1}{d}\left\langle R_{\alpha}(\xi) R_{\alpha}\left(\xi^{\prime}\right)\right\rangle\left\langle R^{2}\left(\xi^{\prime \prime}\right)\right\rangle=\left.\frac{\delta \mathcal{F}\left(\xi, \xi^{\prime}\right)}{\delta Y\left(\xi^{\prime \prime}, \xi^{\prime \prime}\right)}\right|_{0} \\
G\left(\xi, \xi^{\prime}, \xi^{\prime \prime}\right)= & \left.\frac{\delta \mathcal{G}\left(\xi, \xi^{\prime}\right)}{\delta Y\left(\xi^{\prime \prime}, \xi^{\prime \prime}\right)}\right|_{0}, \quad \bar{F}\left(\xi, \xi^{\prime}, \xi^{\prime \prime}\right)=\left.\frac{\delta \overline{\mathcal{F}}\left(\xi, \xi^{\prime}\right)}{\delta Y\left(\xi^{\prime \prime}, \xi^{\prime \prime}\right)}\right|_{0} .
\end{aligned}
$$

Первая величина вьписана подробнее, чем остальные две, поскольку именно она входит в окончательный ответ, в то время как $G\left(\xi, \xi^{\prime}, \xi^{\prime \prime}\right)$ и $\bar{F}\left(\xi, \xi^{\prime}, \xi^{\prime \prime}\right)$ входят лишш в промежуточные вычисления этого неприводимого коррелятора. Нам в дальнейшем понадобятся уравнения на эти корреляторы, выпишем их:

$$
\begin{gathered}
{\left[\partial_{\xi} \partial_{\xi^{\prime}}-\beta g\left(\xi-\xi^{\prime}\right)\right] F\left(\xi, \xi^{\prime}, \xi^{\prime \prime}\right)=} \\
=-\frac{\beta^{2}}{2} \int d \xi_{1} d \xi_{2} g\left(\xi-\xi_{1}\right) g\left(\xi^{\prime}-\xi_{2}\right) F\left(\xi, \xi_{1}\right) F\left(\xi^{\prime}, \xi_{2}\right) \bar{F}\left(\xi_{1}, \xi_{2}, \xi^{\prime \prime}\right), \\
-i \partial_{\xi} G\left(\xi, \xi^{\prime}, \xi^{\prime \prime}\right)+\beta \int d \xi_{1} g\left(\xi-\xi_{1}\right) \bar{F}\left(\xi^{\prime}, \xi_{1}, \xi^{\prime \prime}\right) F\left(\xi, \xi_{1}\right)=0 \\
i \partial_{\xi^{\prime}} G\left(\xi, \xi^{\prime}, \xi^{\prime \prime}\right)+\beta \int d \xi_{1} g\left(\xi^{\prime}-\xi_{1}\right) \bar{F}\left(\xi^{\prime}, \xi_{1}, \xi^{\prime \prime}\right) F\left(\xi, \xi_{1}\right)=2 \delta\left(\xi^{\prime}-\xi^{\prime \prime}\right) F\left(\xi, \xi^{\prime}\right), \\
{\left[\partial_{\xi} \partial_{\xi^{\prime}}-\beta g\left(\xi-\xi^{\prime}\right)\right] \bar{F}\left(\xi, \xi^{\prime}, \xi^{\prime \prime}\right)=-2 \delta\left(\xi-\xi^{\prime \prime}\right) \delta\left(\xi-\xi^{\prime}\right) .}
\end{gathered}
$$

Как видно, эти уравнения даже после ряда упрошаюших предположений оказываются довольно сложными. Они имеют вид неоднородной системы интегро-дифференциальных уравнений и допускают лишь качественное исследование. Ниже приводятся результаты этого исследования в двух предельных случаях $\beta \ll 1$ и $\beta \gg 1$.

А. Короткокоррелированный случай. Случай, когда время корреляции мало по сравнению с характерным временем динамики лагранжевых траекторий, которое оценивается как $D^{-1}$, позволяет аналитически решить написанные выше системы уравнений. Действительно, в этом случае $\beta \ll 1$ и статистику тензора $\sigma_{\alpha \beta}$ можно считать декоррелированной, а все корреляционные функции тензора заменить $\delta$-функциями. После такой замены все уравнения приобретают вид уравнений в частных производных, допускаюших разделение переменных и сведение к обыкновенным дифференциальным уравнениям. Окончательные выражения для тех корреляционных функций, которые понадобятся в дальнейшем при рассмотрении негауссового случая, выписаны ниже:

$$
\begin{gathered}
F\left(\xi_{1}, \xi_{2}\right)=R_{0}{ }^{2} \exp \left[2 \beta \min \left(\xi_{1}, \xi_{2}\right)\right], \\
\bar{F}\left(\xi, \xi^{\prime}, \xi^{\prime \prime}\right)=-2 \exp \left[2 \beta \xi^{\prime \prime}-2 \beta \max \left(\xi, \xi^{\prime}\right)\right] \Theta\left(\xi^{\prime \prime}-\xi\right) \Theta\left(\xi^{\prime \prime}-\xi^{\prime}\right), \\
\bar{\lambda}=D, \quad \Delta=\frac{\bar{\lambda}}{d}=\frac{D}{d} .
\end{gathered}
$$


Б. Долгокоррелированный случай. Приведем результаты для обратного случая, когда флуктуации поля скорости настолько велики, что время корреляции оказывается большим по сравнению с темпом разбегания лагранжевых траекторий. Этот случай отвечает большим значениям параметра $\beta \gg 1$ и оказывается более сложным для исследования. Например, решение для парного коррелятора $F\left(\xi_{1}, \xi_{2}\right)$ можно искать в виде

$$
F\left(\xi_{1}, \xi_{2}\right)=\exp \left(\bar{\lambda} \frac{\xi_{1}+\xi_{2}}{2}\right) \Psi_{0}\left(\xi_{1}-\xi_{2}\right) .
$$

Здесь $\Psi_{0}(\xi)$ - "волновая функция" основного состояния для уравнения Шредингера

$$
\left(\frac{\bar{\lambda}^{2}}{4}-\partial_{\xi}^{2}\right) \Psi_{0}(\xi)=\beta g(\xi) \Psi_{0}(\xi),
$$

a $\bar{\lambda}$ - его энергия. Понятно, что для этого уравнения возможно лишь оценочное исследование. Так как $\beta \gg 1$, то разумно в этом уравнении произвести разложение "потенциала" $g(\xi)$ вблизи точки $\xi=0$ :

$$
g(0)-g(\xi) \propto|\xi|^{a} .
$$

Размер области, в которой функция $\Psi_{0}(\xi)$ сушественно отлична от нуля, можно оценить из соотношения неопределенностей:

$$
\xi_{\text {char }} \propto \beta^{-\frac{1}{2+a}} .
$$

Для коррелятора $\bar{F}$ также можно написать следуюшую оценку:

$$
\bar{F}\left(\xi_{1}, \xi_{2}, \xi\right) \propto \beta^{-\frac{a}{2+a}} \exp \left[\bar{\lambda}\left(\xi-\frac{\xi_{1}+\xi_{2}}{2}\right)\right],
$$

в то время как темп разбегания траекторий имеет вид

$$
\bar{\lambda} \sim \sqrt{\frac{D}{\tau}} .
$$

Подробности получения оценок можно найти в работе [1]

\section{3. РОЛЬ ВЫСШИХ КОРРЕЛЯТОРОВ ГРАДИЕНТА СКОРОСТИ}

Исследуем вклады в $\bar{\lambda}$ и $\Delta$, связанные с высшими неприводимыми корреляторами градиента скорости. При значительном отклонении от гауссовой статистики наша техника перестает работать. Поэтому достаточно рассмотреть эффекты, связанные с неприводимым коррелятором четвертого порядка. Поправки, связанные с более высокими корреляторами, станут сушественными как раз в случае, если $\Delta$ становится порядка $\bar{\lambda}$, т.е. 
на границе области применимости теории. Неприводимый коррелятор четвертого порядка записывается в следуюшем виде:

$$
\begin{aligned}
& \left\langle\left\langle\sigma_{\alpha \beta}\left(t_{1}\right) \sigma_{\gamma \delta}\left(t_{2}\right) \sigma_{\epsilon \mu}\left(t_{3}\right) \sigma_{\rho \nu}\left(t_{4}\right)\right\rangle\right\rangle=\frac{D^{2}}{d^{3} \tau^{2}}\left(h\left(\xi_{1}, \xi_{2} ; \xi_{3}, \xi_{4}\right) \delta_{\alpha \gamma} \delta_{\beta \delta} \delta_{\epsilon \rho} \delta_{\mu \nu}+\right. \\
& \left.\quad+h\left(\xi_{1}, \xi_{3} ; \xi_{2}, \xi_{4}\right) \delta_{\alpha \epsilon} \delta_{\beta \mu} \delta_{\gamma \rho} \delta_{\delta \nu}+h\left(\xi_{1}, \xi_{4} ; \xi_{2}, \xi_{3}\right) \delta_{\alpha \rho} \delta_{\beta \nu} \delta_{\gamma \epsilon} \delta_{\delta \mu}+\cdots\right)
\end{aligned}
$$

где многоточием обозначены члены, несушественные при больших $d$. Теперь мы можем ввести безразмерньй параметр, характеризуюший степень отклонения от гауссовой статистики,

$$
\alpha=\int d \xi_{2} d \xi_{3} d \xi_{4} h\left(0, \xi_{2} ; \xi_{3}, \xi_{4}\right)
$$

При учете неприводимого коррелятора четвертого порядка (45) эффективное действие в главном порядке по $d$ принимает вид

$$
\begin{aligned}
i \mathcal{I}_{\text {eff }}= & i \int d \xi_{1} \overline{\mathbf{R}}_{1} \partial_{t} \mathbf{R}_{1}-\frac{\beta}{2 d} \int d \xi_{1} d \xi_{2} g\left(\xi_{1}-\xi_{2}\right)\left(\overline{\mathbf{R}}_{1} \overline{\mathbf{R}}_{2}\right)\left(\mathbf{R}_{1} \mathbf{R}_{2}\right)+ \\
& +\frac{1}{24} \frac{\beta^{2}}{d^{3}} \int d \xi_{1} d \xi_{2} d \xi_{3} d \xi_{4}\left\{h\left(\xi_{1}, \xi_{2} ; \xi_{3}, \xi_{4}\right)\left(\overline{\mathbf{R}}_{1} \overline{\mathbf{R}}_{2}\right)\left(\overline{\mathbf{R}}_{3} \overline{\mathbf{R}}_{4}\right)\left(\mathbf{R}_{1} \mathbf{R}_{2}\right)\left(\mathbf{R}_{3} \mathbf{R}_{4}\right)+\right. \\
& +h\left(\xi_{1}, \xi_{3} ; \xi_{2}, \xi_{4}\right)\left(\overline{\mathbf{R}}_{1} \overline{\mathbf{R}}_{3}\right)\left(\overline{\mathbf{R}}_{2} \overline{\mathbf{R}}_{4}\right)\left(\mathbf{R}_{1} \mathbf{R}_{3}\right)\left(\mathbf{R}_{2} \mathbf{R}_{4}\right) \times \\
& \left.\times h\left(\xi_{1}, \xi_{4} ; \xi_{2}, \xi_{3}\right)\left(\overline{\mathbf{R}}_{1} \overline{\mathbf{R}}_{4}\right)\left(\overline{\mathbf{R}}_{2} \overline{\mathbf{R}}_{3}\right)\left(\mathbf{R}_{1} \mathbf{R}_{4}\right)\left(\mathbf{R}_{2} \mathbf{R}_{3}\right)\right\}
\end{aligned}
$$

Теперь можно выписать уравнения на парный “коррелятор” $\mathcal{F}$ в главном порядке по $d$ :

$$
\begin{aligned}
& i \partial_{\xi} \mathcal{F}\left(\xi, \xi^{\prime}\right)-\beta \int d \xi_{1} g\left(\xi-\xi_{1}\right) \mathcal{G}\left(\xi_{1}, \xi^{\prime}\right) \mathcal{F}\left(\xi_{1}, \xi\right)+ \\
& \quad+\frac{1}{2} \beta^{2} \int d \xi_{1} d \xi_{2} d \xi_{3} \mathcal{G}\left(\xi_{3}, \xi^{\prime}\right) \overline{\mathcal{F}}\left(\xi_{1}, \xi_{2}\right) \mathcal{F}\left(\xi_{1}, \xi_{2}\right) \mathcal{F}\left(\xi, \xi_{3}\right)\left[h\left(\xi_{1}, \xi_{2} ; \xi_{3}, \xi\right)-\right. \\
& \left.\quad-g\left(\xi_{1}-\xi_{2}\right) g\left(\xi_{3}-\xi\right)\right]+\int d \xi_{1}\left[X\left(\xi, \xi_{1}\right) \mathcal{F}\left(\xi_{1}, \xi^{\prime}\right)+2 Z\left(\xi_{1}, \xi\right) \mathcal{G}\left(\xi^{\prime}, \xi_{1}\right)\right]=0 .
\end{aligned}
$$

Используя тождества, полученные путем дифференцирования эффективного действия (см. (47)), можно получить уравнения и на величины $\mathcal{G}, \overline{\mathcal{F}}$. Но и не проводя этих операций можно увидеть, что использование негауссовой статистики не влияет на вид этих уравнений в предположении, что внешние поля отсутствуют. Действительно, положив $X=Y=Z=0$, получим уравнения на парные корреляторы $F, G$ :

$$
\begin{aligned}
i \partial_{\xi} F\left(\xi, \xi^{\prime}\right)- & \beta \int d \xi_{1} g\left(\xi-\xi_{1}\right) G\left(\xi_{1}, \xi^{\prime}\right) F\left(\xi_{1}, \xi\right)=0 \\
& i \partial_{\xi} G\left(\xi, \xi^{\prime}\right)=\delta\left(\xi-\xi^{\prime}\right) \\
& i \partial_{\xi} G\left(\xi^{\prime}, \xi\right)=-\delta\left(\xi-\xi^{\prime}\right) .
\end{aligned}
$$

Таким образом, мы приходим к выводу, что для парных корреляторов в главном порядке по $d$ негауссовы добавки ничего не дают, так как не изменяют уравнения на эти величины (ср. уравнения (29)- (31)). Это означает, что величина $\bar{\lambda}$ не меняется в рамках рассматриваемого приближения (большие $d$ ). Можно убедиться, что уравнения на 
$\bar{F}\left(\xi, \xi^{\prime}, \xi^{\prime \prime}\right)$ и $G\left(\xi, \xi^{\prime}, \xi^{\prime \prime}\right)$ также не изменяются в главном по размерности пространства порядке. Однако уравнение, определяюшее неприводимый коррелятор $F\left(\xi_{1}, \xi_{2}, \xi_{3}\right)$, претерпевает изменения:

$$
\begin{aligned}
i \partial_{\xi} F\left(\xi, \xi^{\prime}, \xi^{\prime \prime}\right)-\beta \int d \xi_{1} g\left(\xi_{1}-\xi\right)\left[G\left(\xi_{1}, \xi^{\prime}, \xi^{\prime \prime}\right) F\left(\xi_{1}, \xi\right)+G\left(\xi_{1}, \xi^{\prime}\right) F\left(\xi_{1}, \xi, \xi^{\prime \prime}\right)\right]+ \\
+\frac{\beta^{2}}{2} \int d \xi_{1} d \xi_{2} d \xi_{3} h\left(\xi_{3}, \xi ; \xi_{1}, \xi_{2}\right) G\left(\xi_{3}, \xi^{\prime}\right) \bar{F}\left(\xi_{1}, \xi_{2}, \xi^{\prime \prime}\right) F\left(\xi_{3}, \xi\right) F\left(\xi_{1}, \xi_{2}\right)=0 .
\end{aligned}
$$

Дифференцированием получаем уравнение

$$
\begin{aligned}
{\left[\partial_{\xi} \partial_{\xi^{\prime}}-\right.} & \left.\beta g\left(\xi-\xi^{\prime}\right)\right] F\left(\xi, \xi^{\prime}, \xi^{\prime \prime}\right)= \\
= & -\beta^{2} \int d \xi_{1} d \xi_{2} g\left(\xi-\xi_{1}\right) g\left(\xi^{\prime}-\xi_{2}\right) F\left(\xi, \xi_{1}\right) \bar{F}\left(\xi_{1}, \xi_{2}, \xi^{\prime \prime}\right) F\left(\xi_{2}, \xi^{\prime}\right)- \\
& \quad-\frac{\beta^{2}}{2} \int d \xi_{1} d \xi_{2} h\left(\xi^{\prime}, \xi ; \xi_{1}, \xi_{2}\right) \bar{F}\left(\xi_{1}, \xi_{2}, \xi^{\prime \prime}\right) F\left(\xi_{1}, \xi_{2}\right) F\left(\xi, \xi^{\prime}\right) .
\end{aligned}
$$

При выводе этого уравнения было использовано уравнение на $G\left(\xi, \xi^{\prime}, \xi^{\prime \prime}\right)$ (см. [1]). Умножим правую и левую части уравнения $(53)$ на $\bar{F}\left(\xi, \xi^{\prime}, \xi_{3}\right)$ и проинтегрируем по $\xi$ и $\xi^{\prime}$. Воспользовавшись уравнением на коррелятор $\bar{F}\left(\xi, \xi^{\prime}, \xi_{3}\right)$, получим для вклада от неприводимого коррелятора четвертого порядка скорости

$$
\begin{aligned}
F_{h}\left(\xi_{3}, \xi_{3}, \xi^{\prime \prime}\right)= & \frac{\beta^{2}}{4} \int d \xi d \xi^{\prime} d \xi_{1} d \xi_{2} h\left(\xi, \xi^{\prime} ; \xi_{1}, \xi_{2}\right) \times \\
& \times \bar{F}\left(\xi_{1}, \xi_{2}, \xi^{\prime \prime}\right) F\left(\xi_{1}, \xi_{2}\right) \bar{F}\left(\xi, \xi^{\prime}, \xi_{3}\right) F\left(\xi, \xi^{\prime}\right) .
\end{aligned}
$$

Произведем оценку интеграла. Для этого вспомним, как ведут себя функции $F\left(\xi_{1}, \xi_{2}\right)$ и $\bar{F}\left(\xi_{1}, \xi_{2}, \xi^{\prime \prime}\right)$. Обе эти функции в пределе $\beta \gg 1$ сильно спадают при $\xi_{1}-\xi_{2} \approx \xi_{\text {char }}$, поэтому в $h\left(\xi, \xi^{\prime} ; \xi_{1}, \xi_{2}\right)$ можно положить $\xi \approx \xi^{\prime}$ и $\xi_{1} \approx \xi_{2}$. В результате интегрирования по разностям $\xi-\xi^{\prime}, \xi_{1}-\xi_{2}$ получим $\xi_{\text {char }}^{2}$, по разности $\xi-\xi_{1}$ - параметр $\alpha$, а четвертое интегрирование даст $\min \left(\xi_{3}, \xi^{\prime \prime}\right)$. В итоге приходим к результату:

$$
F_{h}\left(\xi, \xi, \xi^{\prime \prime}\right) \propto \alpha \beta^{\frac{a+3}{a+2}} \min \left(\xi, \xi^{\prime \prime}\right) \exp \left(\bar{\lambda}\left(\xi+\xi^{\prime \prime}\right)\right) .
$$

В то же время

$$
F(\xi, \xi)=\Psi_{0}(0) e^{\bar{\lambda} \xi} \sim \beta^{\frac{1}{2(a+2)}} e^{\bar{\lambda} \xi} .
$$

Теперь можно определить дисперсию:

$$
\Delta_{\mathrm{LR}} \propto \frac{\alpha}{d \tau} \beta .
$$

Рассмотрим противоположный случай короткокоррелированного поля скорости: $\beta \ll 1$. В этом случае корреляционные функции тензора $\sigma_{\alpha \beta}$ заменяются на выражения типа

$$
\begin{aligned}
g(\xi) & =2 \delta(\xi), \\
h\left(\xi_{1}, \xi_{2} ; \xi_{3}, \xi_{4}\right) & =\frac{\alpha}{d} \delta\left(\xi_{1}-\xi_{2}\right) \delta\left(\xi_{3}-\xi_{4}\right) \delta\left(\xi_{2}-\xi_{3}\right) .
\end{aligned}
$$

6 Теоретическая и математическая физика, т. 122, № 3, 2000 г. 
Воспользовавшись тем фактом, что корреляторы, стояшие под интегралом (54), не изменились в пределе больших размерностей пространства $d$ под влиянием отклонения статистики скорости от гауссовой, несложно вычислить упомянутый интеграл, подставив в него уже известные выражения:

$$
F_{h}\left(\xi, \xi, \xi^{\prime}\right)=R_{0}^{4} \alpha \beta^{2} \exp \left[2 \beta\left(\xi+\xi^{\prime}\right)\right] \min \left(\xi, \xi^{\prime}\right)
$$

В этом случае коррелятор (16) имеет вид

$$
\frac{\left\langle\left\langle R^{4}\right\rangle\right\rangle}{\left\langle R^{2}\right\rangle^{2}}=\frac{d F_{h}(\xi, \xi, \xi)}{d^{2} F(\xi, \xi)^{2}}=\frac{\alpha \beta^{2}}{d \tau} t .
$$

Отсюда непосредственно находим дисперсию:

$$
\Delta_{\mathrm{SR}}=\frac{\alpha D^{2} \tau}{4 d}
$$

Полезно переписать полученные в двух предельных случаях выражения для дисперсии через средний темп разбегания:

$$
\begin{aligned}
& \Delta_{\mathrm{LR}} \propto \frac{\alpha \bar{\lambda}^{2} \tau}{d}, \\
& \Delta_{\mathrm{SR}}=\frac{\alpha \bar{\lambda}^{2} \tau}{4 d} .
\end{aligned}
$$

\section{4. ЗАКЛЮЧЕНИЕ}

Мы исследовали статистику расстояния между лагранжевыми траекториями $R$ в крупномасштабном поле скорости. На временах, бо́льших времени корреляции скорости, мы получили логнормальную статистику, которой соответствует выражение (10). Используя технику больших размерностей пространства $d$, мы оценили влияние различных параметров, характеризуюших статистику градиентов скорости, на величины $\bar{\lambda}$ и $\Delta$, входящие в (10). Имеются два основных безразмерных параметра: $\beta(22)$, определяющий время корреляции градиентов скорости, и $\alpha(46)$, определяющий степень отклонения статистики градиентов скорости от гауссовой. Зависимость $\bar{\lambda}$ и $\Delta$ от $\beta$ исследовалась в работе [1], где было показано, что $\bar{\lambda} \sim D$ при $\beta \ll 1$ и $\bar{\lambda} \sim \sqrt{D / \tau}$ при $\beta \gg 1$. При малых $\beta$ оценка $\Delta$ универсальна: $\Delta \sim D$, в то время как при больших $\beta$ она зависит от показателя $a($ см. $(41)): \Delta \sim \beta^{(1+a) /(2+a)} / \tau$. Неприводимый коррелятор четвертого порядка градиентов скорости (45) дает дополнительный вклад в $\Delta$, который можно оценить как $\alpha D^{2} \tau$ при $\beta \ll 1$ и $\alpha \beta / \tau$ при $\beta \gg 1$. Можно ввести критическое значение $\alpha_{\mathrm{cr}}$ параметра $\alpha$, начиная с которого роль высших неприводимых корреляторов градиента скорости становится определяющей. Это критическое значение может быть оценено:

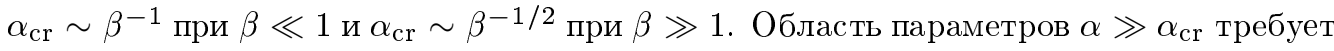
особого рассмотрения, так как наша техника в этой области не работает.

Благодарности. Автор выражает свою признательность В.В.Лебедеву, сыгравшему руководящую роль при написании этой статьи. 


\section{Список литературы}

[1] G. Falkovich, V. Kazakov, V. Lebedev. Physica A. 1998. V. 249. P. 36.

[2] L. Richardson. Proc. Roy. Soc. London A. 1926. V. 110. P. 709.

[3] R. H. Kraichnan. Phys. Fluids. 1964. V. 7. P. 1723; 1965. V. 8. P. 575.

[4] B. Shraiman, E. Siggia. Phys. Rev. E. 1994. V. 49. P. 2912.

[5] R. H. Kraichnan. Phys. Fluids. 1966. V. 9. P. 1937.

[6] M. Lesieur. Turbulence in Fluids. London: Kluwer Acad. Publ., 1990.

[7] R. H. Kraichnan. Phys. Fluids. 1968. V. 11. P. 945.

[8] R. H. Kraichnan. J. Fluid Mech. 1974. V. 64. P. 737.

[9] G. Falkovich, I. Kolokolov, V. Lebedev, A. Migdal. Phys. Rev. E. 1996. V. 54. P. 4896.

[10] G. K. Batchelor. J. Fluid Mech. 1959. V. 5. P. 113.

[11] M. Chertkov, G. Falkovich, I. Kolokolov, V. Lebedev. Phys. Rev. E. 1995. V. 51. P. 5609.

[12] M. Chertkov, A. Gamba, I. Kolokolov. Phys. Lett. A. 1994. V. 192. P. 435.

[13] G. 't Hooft. Nucl. Phys. B. 1974. V. 72. P. 461.

[14] H. E. Stanley. Phys. Rev. 1968. V. 176. P. 718.

[15] H.W. Wyld. Ann. Phys. 1961. V. 14. P. 143.

[16] P. C. Martin, E. D. Siggia, H. A. Rose. Phys. Rev. A. 1973. V. 8. P. 423.

[17] S. K. Ma. Modern Theory of Critical Phenomena. New York: Benjamin, 1976.

[18] C. de Dominicis. J. Phys. (Paris). Suppl. Colloque C-1. 1976. V. 37. P. 247.

[19] H. K. Janssen. Z. Phys. B. 1976. V. 23. P. 377.

[20] B. Н. Попов. Функциональные интегралы в квантовой теории поля и статистической физике. М.: Атомиздат, 1976.

Поступила в редакцию 9.IV.1999 г., после доработки 28.VI.1999 г. 